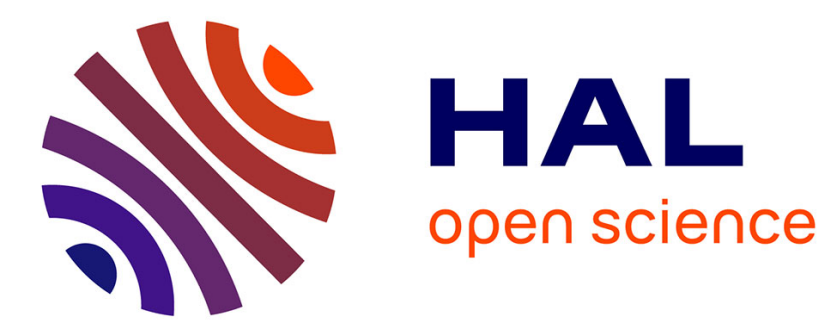

\title{
Conversion de modes de Lamb à l'extrémité d'une plaque immergée
}

\author{
M. Ech-Cherif El-Kettani, P. Pareige, F. Luppé
}

\section{To cite this version:}

M. Ech-Cherif El-Kettani, P. Pareige, F. Luppé. Conversion de modes de Lamb à l'extrémité d'une plaque immergée. Journal de Physique IV Proceedings, 1994, 04 (C5), pp.C5-873-C5-876. 10.1051/jp4:19945190 . jpa-00252873

\section{HAL Id: jpa-00252873 https://hal.science/jpa-00252873}

Submitted on 1 Jan 1994

HAL is a multi-disciplinary open access archive for the deposit and dissemination of scientific research documents, whether they are published or not. The documents may come from teaching and research institutions in France or abroad, or from public or private research centers.
L'archive ouverte pluridisciplinaire HAL, est destinée au dépôt et à la diffusion de documents scientifiques de niveau recherche, publiés ou non, émanant des établissements d'enseignement et de recherche français ou étrangers, des laboratoires publics ou privés. 


\title{
Conversion de modes de Lamb à l'extrémité d'une plaque immergée
}

\author{
M. ECH-CHERIF EL-KETTANI, P. PAREIGE et F. LUPPÉ \\ LAUE, URA 1373 du CNRS, Université du Havre, place Robert Schuman, 76610 Le Havre, France
}

\begin{abstract}
Résumé: This paper deals with an experimental study of the conversion of an incident Lamb wave on the extremity of an immersed plate. This wave gives rise to one or several different Lamb waves propagating in the opposite direction. The converted waves are observed only after a distance from the end of the plate. Non guided waves are also observed backward in the plate. An interpretation is proposed, based on the description of Lamb waves by the Debye series.
\end{abstract}

\section{Introduction.}

Plusieurs phénomènes se produisent quand une onde de Lamb incidente rencontre l'extrémité d'une plaque immergée. La conversion en ondes de volume dans l'eau et la rétroréflexion (réflexion de l'onde incidente en elle-même) ont déjà été étudiées [1,2]. L'objet de cet article est l'étude expérimentale de la conversion de l'onde de Lamb incidente en des ondes de natures différentes se propageant en retour dans la plaque. L'existence de ces ondes est justifiée théoriquement par le fait que les conditions de continuité des contraintes dans la direction normale à l'extrémité de la plaque ne peuvent être satisfaites par la seule présence de l'onde incidente et de l'onde rétroréfléchie [3]. A notre connaissance, peu d'études expérimentales précises ont été consacrées au sujet [4].

\section{Etude expérimentale.}

\subsection{Dispositif expérimental.}

L'étude expérimentale consiste à générer une onde de Lamb incidente se propageant dans la direction de l'extrémité de la plaque et d'observer l'ensemble des ondes issues de leur interaction et se propageant dans le sens opposé. Par conséquent, l'étude est réalisée en régime quasi-harmonique. La géométrie de l'expérience est donnée sur la figure 1 . La plaque étudiée est en aluminium d'épaisseur $\mathrm{d}=5 \mathrm{~mm}$. Deux transducteurs de même fréquence centrale $(2,25 \mathrm{MHz})$ sont utilisés, l'un fonctionnant en émetteur et l'autre en récepteur. Le transducteur émetteur insone la plaque sous un angle d'incidence $\theta_{\mathrm{i}}$ choisi, générant ainsi une onde de Lamb. L'étude concerne les modes de Lamb situés sur la verticale des courbes de dispersion à un produit fréquence-épaisseur $\mathrm{fd}=6,5 \mathrm{kHz} \mathrm{m}$. Le transducteur récepteur est orienté sous un angle $\theta_{\mathrm{r}}$ variant de 0 à 30 degrés, et peut être translaté parallèlement à la plaque. 


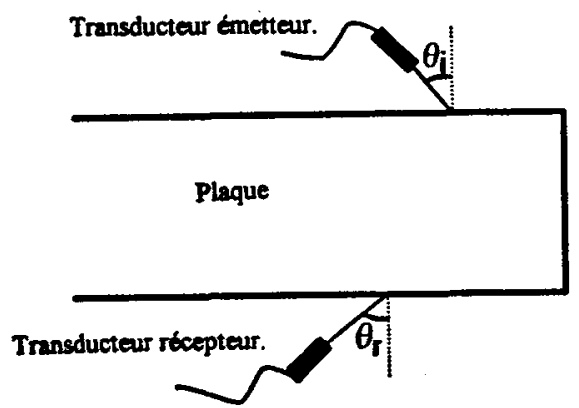

Figure 1: Gtometrie de l'expérience.

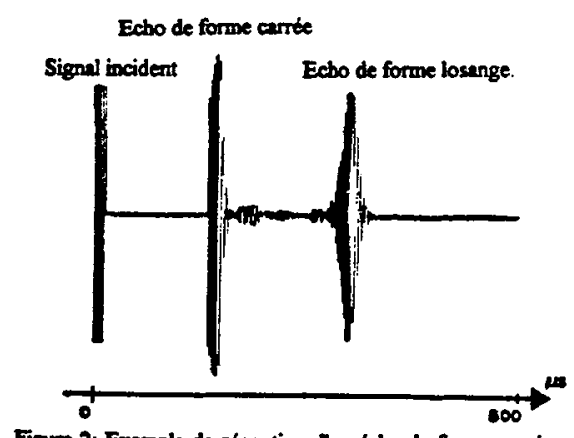

Figure 2: Exernple de recption dun bcho de forme cartic et oun beho de forme loange. Mode incident $\mathrm{S}_{3}$, fot $-6,5 \mathrm{kHz} \mathrm{m}$.

\subsection{Résultats et analyse.}

Quel que soit le mode incident, deux types d'échos sont observés. Ils se distinguent par leur forme carrée ou losange. Pour des angles de réception $\theta_{\mathbf{r}}$ différents de l'angle de réémission d'une onde de Lamb, seuls les échos de forme carrée sont observés. Les échos de forme losange, quand ils sont observés, sont reçus sous un angle $\theta_{\mathrm{r}}$ correspondant à l'angle de réémission d'une onde de Lamb et sont parfois observés avec des échos de forme carrée. Un exemple est donné sur la figure 2. Pour pouvoir juger du caractère propagatif des ondes associées à ces échos, une étude en translation parallèlement à la plaque est effectuée.

\subsubsection{Etude des échos de forme carrée.}

Nous avons choisi pour cette étude un produit fréquence-épaisseur tel qu'une seule onde de Lamb puisse être observable. Nous avons donc généré, dans la direction de l'extrémité d'une plaque d'aluminium d'épaisseur $\mathrm{d}=0,5 \mathrm{~mm}$, le mode $\mathrm{S}_{0}$ à $\mathrm{fd}=1 \mathrm{kHz} \mathrm{m}$. Pour une position angulaire $\theta_{\mathrm{r}}$ supérieure à l'angle d'excitation du mode $S_{0}\left(\cong 16^{\circ}\right)$, un suivi de l'écho reçu est effectué par translation du transducteur récepteur sur toute la longueur de la plaque. Quel que soit l'angle de réception $\theta_{\mathrm{r}}$ compris dans l'intervalle d'étude, les échos de forme carrée sont systématiquement observés. Leur apparition n'intervient qu'à partir d'une certaine distance de l'extrémité de la plaque. Cette distance augmente avec l'angle $\theta_{\mathbf{r}}$. Nous avons reporté sur la figure 3 la zone d'observabilité de ces échos en fonction de $\theta_{\mathbf{r}}$. Leur amplitude n'a aucune évolution régulière en fonction de la distance de propagation (amortissement en fonction de la distance de propagation). Nous en concluons que ces échos ne correspondent pas à des ondes guidées.

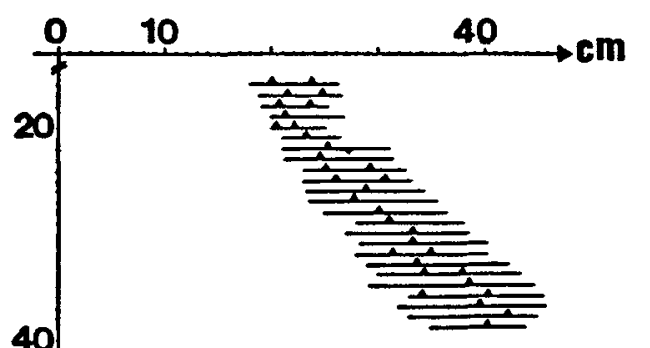

Figure 3: Zone d'observabilité des echos de forme carté en fonction de langle de réception. Les distances sont mesurces a partir de lextrémité de la plaque. Les triangles indiquent in position où lamplitude de ces échos est maximale.

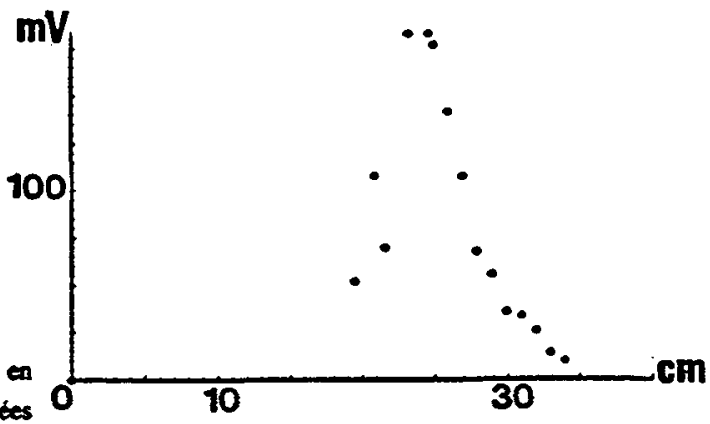

Figure 4: Evohution de ramplitude din beho de forme losange en fonction de la distance par rapport rextrémité de la plaque. Mode incident $\mathrm{S}_{3}$, reception cous l'angle de retmission du mode $A_{1}, f d=6,5 \mathrm{kHz}$. 


\subsubsection{Etude des échos de forme losange.}

Les échos de forme losange, caractéristiques d'ondes guidées [5], sont observés pour un angle de réception $\theta_{\mathrm{r}}$ correspondant à l'angle de réémission d'un mode de Lamb. Ces échos ne sont observés qu'à partir d'une certaine distance de l'extrémité de la plaque. Pour un mode incident donné, cette distance augmente avec l'angle $\theta_{\mathbf{r}}$. Pour un angle $\theta_{\mathbf{r}}$ donné, cette distance est constante quel que soit le mode incident. Afin de vérifier que ces échos correspondent à des modes de Lamb convertis, l'évolution de leur amplitude a été suivie en fonction de la distance par rapport à l'extrémité de la plaque. La figure 4 présente cette évolution pour l'écho reçu sous l'angle $\theta_{\mathrm{r}}$ caractéristique du mode $A_{1}$ (mode $S_{3}$ incident). La distance d'apparition est ici de l'ordre de $18 \mathrm{~cm}$. A partir de cette distance, l'amplitude subit des oscillations avant d'atteindre un maximum à $23 \mathrm{~cm}$. Nous assistons alors à une décroissance régulière de l'amplitude de l'écho jusqu'à annulation de celui-ci. A partir d'une distance $\mathrm{L}$, cette décroissance suit une variation exponentielle. Il est tentant d'attribuer le nom de mode de Lamb converti à toutes les ondes associées à ce type d'écho. Cependant, nous nous sommes imposés un critère restrictif. Une mesure d'atténuation [6] est systématiquement effectuée dans la zone où leur amplitude décroît d'une façon exponentielle. Seuls les échos dont l'atténuation est identique à celle du mode de Lamb réémettant sous le même angle $\theta_{\mathrm{r}}$ et qui se propagerait dans une plaque de longueur infinie, sont considérés comme effectivement convertis. La figure 5 représente de façon schématique les conversions vérifiant ce critère, pour $\mathrm{fd}=6,5 \mathrm{kHz} \mathrm{m}$. Cette figure montre que tout mode incident donne naissance à des modes convertis, à l'exception du mode $\mathrm{A}_{2}$. Nous remarquons également que les modes $A_{1}$ et $S_{1}$ sont le plus souvent observés en conversion. Ceci peut être lié au fait que ces modes présentent une forte dominante transversale. Cependant c'est également le cas du mode $A_{2}$ qui pourtant n'est observé qu'une seule fois en conversion.
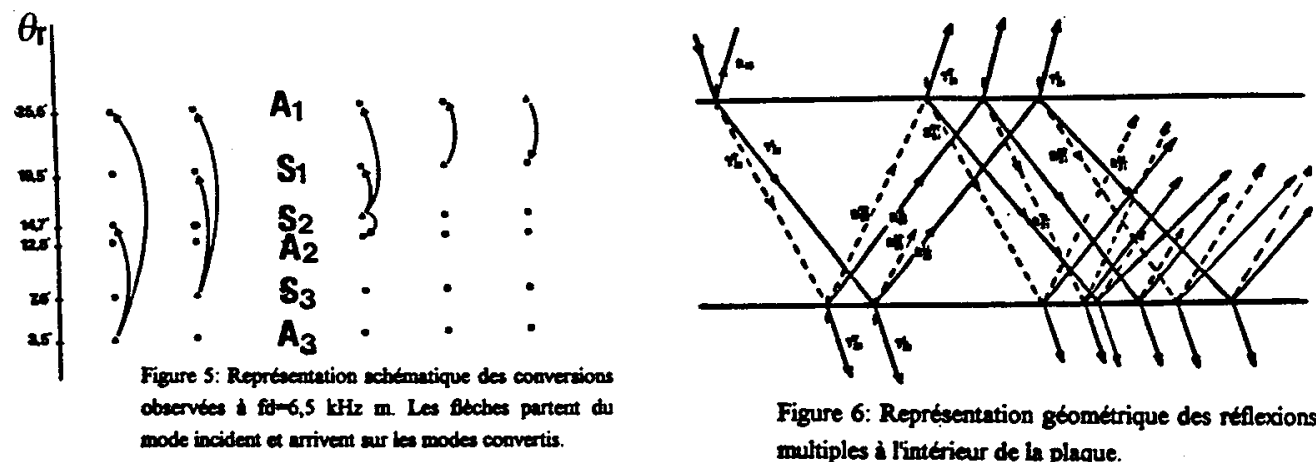

Figure 6: Reprécentation gtometrique des reflexions multiples ì lintérieur de la plaque.

\section{Interprétation.}

L'examen de la figure 4 donne l'impression que le mode converti prend progressivement naissance dans la plaque $(x<25 \mathrm{~cm})$. Pour interpréter ce résultat, nous avons pensé que ce mode est construit petit à petit par des réflexions multiples d'ondes longitudinales et transversales issues de l'extrémité de la plaque. Ceci est analogue à la description des ondes de Lamb en termes de série de Debye [7] dont la figure 6 donne une représentation géométrique. La série converge quand un nombre $\mathrm{N}$ suffisant d'interactions est pris en compte, ce qui revient à dire que le mode de Lamb n'est formé qu'à partir d'une certaine distance $\mathrm{D}$ nécessaire à l'obtention de ce nombre d'interactions. Nous avons donc effectué une étude numérique de la convergence de la série de Debye afin de déterminer N. Pour chacun des modes de Lamb étudiés, nous avons tracé le module du coefficient de réflexion exact en fonction de la fréquence, et nous l'avons 
comparé à celui obtenu par la série de Debye en sommant un nombre de termes $\mathrm{N}$ variable. Nous constatons, pour $\mathrm{N}=10$, une bonne concordance entre les deux coefficients pour tous les modes, excepté le mode $S_{3}$ et, dans une moindre mesure, $S_{2}$ (Un exemple est donné sur la figure 7 pour le mode $S_{1}$ ). Ces résultats sont à rapprocher de ceux résumés sur la figure 5 . Nous constatons sur cette figure que tous les modes ont été observés au moins une fois en conversion, à l'exception du mode $\mathrm{S}_{3}$. Le cas du mode $\mathrm{A}_{3}$ est à considérer à part, car même s'il peut être obtenu en conversion, la valeur très élevée de son coefficient d'amortissement $\left(140 \mathrm{~m}^{-1}\right)$ en rendrait toute observation impossible. Nous avons calculé la distance $D$ pour les modes convertis $A_{1}$ et $S_{1}$ (qui sont à forte dominante transversale), en considérant que les dix interactions nécessaires à leur obtention, correspondent uniquement à des ondes transversales. La valeur obtenue (quelques dizaines de centimètres) donne un ordre de grandeur comparable à la distance d'observation expérimentale $\mathrm{L}$.

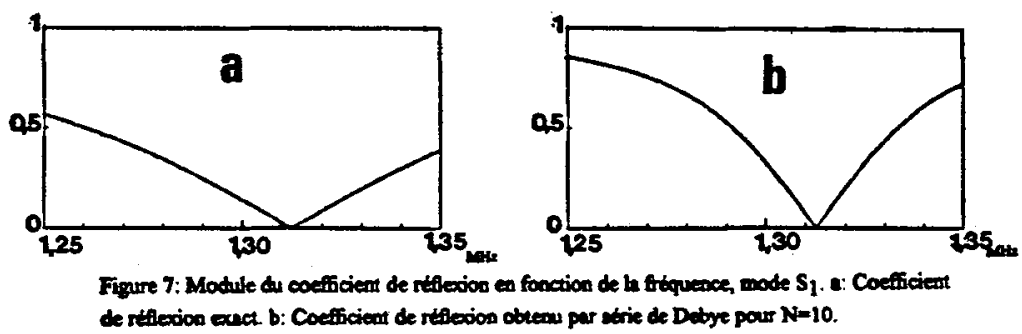

\section{Conclusion.}

Nous avons observé, en retour dans la plaque, des ondes de Lamb converties, différentes de l'onde incidente, ainsi que des ondes non guidées. Ces ondes n'apparaissent qu'à partir d'une certaine distance de l'extrémité de la plaque. L'aspect propagatif des ondes de Lamb converties a été mis en évidence par la mesure de leur coefficient d'amortissement. L'étude de la convergence de la série de Debye pour les modes convertis a permis d'obtenir un ordre de grandeur de la distance d'apparition d'une onde convertie à polarisation transversale. La poursuite de l'étude de la conversion est en cours. L'objectif est de voir s'il est possible d'établir un lien entre une onde convertie et sa polarisation, et si l'analyse par la série de Debye peut être un moyen d'investigation systématique.

\section{Bibliographie:}

[1] P. Pareige, F. Luppé, J. Ripoche, "Scattering of Lamb waves at the edge of a semi-infinite plate embedded in water", J. Acoust. Soc. Am. 92, pp 1056-1060 (1992)

[2] M. Ech-Cherif El-Kettani, P. Pareige, F. Luppé, "Scattering by a semi-infinite elastic plate immersed in water", 2 ème Congrès Français d'Acoustique, Arcachon, 1991, acte dans J. Phys. IV

[3] e.g. Peter J. Torvik, "Reflection of wave trains in semi-infinite plates.", J. Acous. Soc. Am. 41, pp 346353 (1967)

[4] P. Diodati, G. Tassi, "Lamb wave reflection at plate edges", Appl. Phys. Lett. 47, pp 573-575 (1985)

[5] J.-L. Izbicki, G Maze, J. Ripoche, "Etude de la réémission libre d'une plaque résonante",Acustica 55, pp 27-29 (1984)

[6] M. Ech-Cherif El Kettani, P. Pareige, F. Luppé, "Experimental determination of the evanescent coefficient of leaking Lamb waves", soumis à Acoust. Lett.

[7] J. M. Conoir, A. Gérard, A. Derem, "Ondes acoustiques transmises et séries de Debye généralisées. 1 Traitement des interfaces planes", J. Acoustique 4, pp 159-200 (1991) 